\title{
Assessment of myocardial viability using F-18 fluorodeoxyglucose/Tc-99m sestamibi dual-isotope simultaneous acquisition SPECT: Comparison with Tl-201 stress-reinjection SPECT
}

\author{
Yen-Wen Wu, MD, a Por-Jau Huang, MD, ${ }^{\mathrm{b}}$ Chii-Ming Lee, $M D$, PhD, ${ }^{\mathrm{b}}$ \\ Yi-Lwun Ho, MD, PhD, ${ }^{\mathrm{b}}$ Lung-Chun Lin, MD, ${ }^{\mathrm{b}}$ Tzung-Dau Wang, MD, PhD, ${ }^{\mathrm{b}}$ \\ Shoei-Shen Wang, MD, PhD, ${ }^{c}$ Tony Hsiu-Hsi Chen, PhD, ${ }^{\mathrm{d}}$ and Ruoh-Fang Yen, $\mathrm{MD}^{\mathrm{a}, \mathrm{d}}$
}

Background. This study compared technetium 99m sestamibi/fluorine 18 fluorodeoxyglucose dual-isotope simultaneous acquisition (DISA) with stress-reinjection thallium 201 single photon emission computed tomography (SPECT) with regard to their ability to detect myocardial viability.

Methods and Results. The study cohort consisted of 42 angiographically significant coronary artery disease patients with symptomatic congestive heart failure or regional wall motion abnormalities. In total, 398 dysfunctional segments in 40 patients were analyzed ( 2 patients were excluded because of poor-quality F-18 fluorodeoxyglucose images). Of the segments, 217 were diagnosed as viable and 144 as nonviable by both DISA and Tl-201, 33 were viable by DISA but nonviable by Tl-201, and 4 were viable by Tl-201 but nonviable by DISA. Most discrepancies were in the inferior wall. Of the 40 patients, 16 underwent revascularization. From the follow-up results for the $\mathbf{1 0 5}$ dysfunctional segments in these 16 patients, DISA viability appears to be a significant predicting factor $(P=.014)$ for functional recovery after revascularization statistically whereas $\mathrm{Tl}-201$ viability does not $(P=.09)$.

Conclusion. Our study suggests that DISA viability provides more accurate prediction of postrevascularization functional recovery than Tl-201 viability. Given the small number of patients who underwent revascularization, the superiority of DISA over Tl-201 in detecting myocardial viability may be firmly established by further study on a large scale for patients with profound left ventricular dysfunction. (J Nucl Cardiol 2005;12:451-9.)

Key Words: Myocardial viability • dual-isotope simultaneous acquisition • single photon emission computed tomography • fluorine 18 fluorodeoxyglucose • technetium 99m sestamibi

It has been well documented that the numbers of patients with chronic heart failure due to coronary artery disease have increased rapidly ${ }^{1}$ and that patients with myocardial viability and impaired left ventricular (LV) function can benefit from coronary revascularization. ${ }^{2,3}$

From Departments of Nuclear Medicine, ${ }^{\mathrm{a}}$ Internal Medicine, ${ }^{\mathrm{b}}$ and Surgery, ${ }^{\mathrm{c}}$ National Taiwan University Hospital and National Taiwan University College of Medicine, and Institute of Preventive Medicine, National Taiwan University College of Public Health, ${ }^{\mathrm{d}}$ Taipei, Taiwan.

The work was supported in part by grant NSC-90-2314-B-002-210 from the National Science Council, Taiwan.

Received for publication Dec 7, 2004; final revision accepted Mar 24, 2005

Reprint requests: Ruoh-Fang Yen, MD, Department of Nuclear Medicine, National Taiwan University Hospital, No. 7, Chung-Shan South Road,

Taipei, Taiwan; rfyen@ha.mc.ntu.edu.tw.

$1071-3581 / \$ 30.00$

Copyright $\odot 2005$ by the American Society of Nuclear Cardiology.

doi:10.1016/j.nuclcard.2005.04.007
Currently, among the various techniques that have been introduced to assess myocardial viability, ${ }^{4,5}$ positron emission tomography (PET) by use of fluorine 18 fluorodeoxyglucose (FDG) as a metabolic tracer and nitrogen 13 ammonia as a perfusion tracer has emerged as the gold standard for such assessment. ${ }^{6-8}$ However, the high cost of the PET equipment and the requirement of an onsite cyclotron for production of short-life PET perfusion tracers have limited the scope of its clinical applications. $^{9}$

Recently, F-18 FDG cardiac images obtained with a single photon emission computed tomography (SPECT) camera and 511-keV ultrahigh-energy collimators have been shown to provide clinically relevant data equivalent to those obtained with PET. ${ }^{10-13}$ Delbeke and colleagues ${ }^{14,15}$ have demonstrated the feasibility in analyzing myocardial perfusion/metabolism simultaneously by use of technetium 99m sestamibi (MIBI)/F-18 FDG with a dual-isotope simultaneous acquisition (DISA) protocol. 
The objective of this study was to compare Tc- $99 \mathrm{~m}$ MIBI/F-18 FDG DISA with stress-reinjection thallium 201 SPECT with regard to their ability to effectively detect myocardial viability.

\section{METHODS}

\section{Patient Population}

We recruited 42 patients with angiographically significant coronary artery disease $(\geq 70 \%$ diameter stenosis) (39 men with mean weight of $66.9 \pm 6.0 \mathrm{~kg}$ and 3 women with mean weight of $50.7 \pm 5.1 \mathrm{~kg}$ ), aged 34 to 85 years, with chronic congestive heart failure (New York Heart Association [NYHA] functional class II or higher) or regional wall motion abnormalities on resting echocardiography. No patient had had recent ( $<6$ weeks) myocardial infarction, unstable angina pectoris, or concomitant valve disease. Eleven patients had diabetes mellitus. All patients received DISA and stress-reinjection Tl-201 SPECT within 1 week of each other. Written informed consent was obtained from all patients.

\section{TC-99m MIBI/F-18 FDG DISA SPECT}

Nondiabetic patients received $50 \mathrm{~g}$ of glucose orally after fasting for at least 4 hours, whereas diabetic patients were administered soluble insulin intravenously according to their respective blood glucose levels. ${ }^{16}$ Sixty minutes later, each patient was injected with 6 to $8 \mathrm{mCi}$ F-18 FDG and $20 \mathrm{mCi}$ Tc-99m MIBI. After 60 minutes of uptake phase, each patient underwent DISA ${ }^{14}$ performed on a Hawkeye (GE Medical Systems, Milwaukee, Wis) equipped with 5/8-inch-thick sodium iodide (thallium) (NaI[Tl]) crystals and 511-keV ultrahigh-energy high-resolution collimators. The resolution of the system was $8.6 \mathrm{~mm}$ at $140 \mathrm{keV}$ and $11.8 \mathrm{~mm}$ at $511 \mathrm{keV}$ in full width at half maximum. Two energy windows were set at 140 $\mathrm{keV}( \pm 10 \%)$ of $\mathrm{Tc}-99 \mathrm{~m}$ and at $511 \mathrm{keV}( \pm 10 \%)$ of F-18. Thirty-two projections were acquired over a circular orbit of $180^{\circ}\left(45^{\circ}\right.$ right anterior oblique to $45^{\circ}$ left posterior oblique at 60 seconds per angle). The contribution to the Tc-99m window from downscatter caused by the presence of F-18 was insignificant. ${ }^{17}$ Transaxial slices, $2.8 \mathrm{~mm}$ in thickness ( 1 pixel), were reconstructed by ordered-subset expectation maximization from the raw data collected by use of a Butterworth filter. Simultaneous reconstruction of the $140-$ and $511-\mathrm{keV}$ data sets allowed direct comparison of exactly aligned perfusion and metabolism images.

\section{Stress-Reinjection TI-201 SPECT}

Nineteen patients were studied by use of the symptom-limited modified Bruce exercise protocol ${ }^{18}$ with 3 mCi Tl-201 injected at peak exercise. For those who were unable to exercise adequately, we used pharmacologic stress testing ${ }^{19,20}$; dipyridamole was used in 16 patients, and dobutamine was used in the remaining 7 patents, who had a history of chronic obstructive pulmonary disease.

Stress imaging was started within 5 minutes of the completion of the stress test, and redistribution imaging was acquired 4 hours later. Tl-201 SPECT was performed by use of a dual-head gamma camera (DST-XL; SMV, Buc, France) equipped with 3/8-inch-thick $\mathrm{NaI}(\mathrm{Tl})$ crystals and low-energy high-resolution collimators. The resolution of the system was 7.5-mm full width at half maximum at $120 \mathrm{keV}$. Two energy windows were set at $72 \mathrm{keV}( \pm 10 \%)$ and at $167 \mathrm{keV}( \pm 10 \%)$ of Tl-201. Thirty-two projections were acquired from $45^{\circ}$ right anterior oblique to $45^{\circ}$ left posterior oblique at 30 seconds per angle. Images were reconstructed by use of filtered backprojection with a Butterworth filter (cutoff, 0.5 ; order, 4) and reoriented to obtain standard shortaxis, horizontal long-axis, and vertical long-axis images of the heart.

If the resulting images showed irreversible defects, additional Tl-201 $(1 \mathrm{mCi})$ was injected and a third set of images was obtained 15 minutes after reinjection.

\section{Image Interpretation and Data Analysis}

Attenuation correction for both DISA and Tl-201 SPECT was not used in this study. SPECT images were normalized such that peak myocardial activity was $100 \%$. All images were interpreted qualitatively by 2 experienced nuclear medicine physicians who had no previous knowledge of any patient's clinical data. In the event that the 2 interpretations did not agree with one another, the image was reevaluated by the 2 physicians until a consensus was reached. The left ventricle was divided into 17 segments for analysis. ${ }^{21}$

For both DISA and Tl-201 SPECT, tracer activity in each segment was scored as follows: 3, normal (activity $>70 \%$ of peak activity); 2 , mildly reduced $(51 \%-70 \%)$; 1 , moderately reduced (30\%-50\%); and 0 , severely reduced $(<30 \%)$. Segments with marked reduction of both F-18 FDG and Tc-99m MIBI (score of 0 or 1) were classified as DISA-nonviable. Segments with marked reduction of Tc-99m MIBI but normal or mildly reduced uptake of F-18 FDG (score of 2 or 3) were classified as DISA-viable. ${ }^{22-24}$

For Tl-201 SPECT, stress images were compared with redistribution and reinjection images to distinguish 
Table 1. Patient profiles $(\mathrm{N}=40)$

\begin{tabular}{lc}
\hline \multicolumn{1}{c}{$\begin{array}{c}\text { Clinical } \\
\text { characteristics }\end{array}$} & Finding \\
\hline Age (y) (range) & $61.3 \pm 13.0(34-85)$ \\
Male gender & $37(92.5 \%)$ \\
Hypertension & $16(40 \%)$ \\
Diabetes mellitus & $11(26.2 \%)$ \\
Hyperlipidemia & $10(25 \%)$ \\
LVEF (\%) (range) & \\
Prior cardiac events & $21(52.5 \%)$ \\
Previous revascularization & $26(67.5 \%)$ \\
Previous MI & $11(27.5 \%)$ \\
Q-wave MI & \\
\hline
\end{tabular}

MI, Myocardial infarction.

reversible defects from irreversible defects. A myocardial segment with a severe perfusion defect (score of 0 or 1) was considered nonviable if the defect was irreversible or minimally reversible (Tl-201 uptake in the reinjection images still $<50 \%$ ). A defected segment was considered to be viable if it was reversible (Tl-201 uptake in the redistribution or reinjection images $\geq 50 \%)^{20,25-27}$

\section{Echocardiography}

All patients had undergone resting 2-dimensional echocardiography to assess myocardial contractile function by use of a Sonos-4500/5500 imaging system (Hewlett-Packard, Palo Alto, Calif) with harmonic imaging. Standard parasternal long- and short-axis slices were obtained, as well as apical long-axis 2- and 4-chamber views, as recommended by the American Society of Echocardiography. ${ }^{21,28}$ In each analysis the left ventricle was divided into 17 segments and each segment was visually scored by 2 experienced observers who were unaware of the patients' angiographic and scintigraphic results. A 5-point scoring system (1, normokinesia; 2, mild hypokinesia; 3 , moderate or severe hypokinesia; 4 , akinesia; and 5, dyskinesia) was used. LV ejection fraction (EF) was measured by the area-length method. The consensus rate for interobserver agreement with regard to the echocardiographic findings in this study was $94.2 \%$, which is consistent with previously reported results from our center. ${ }^{29}$

\section{Follow-up Assessment}

Of the 42 patients in this study, 16 underwent revascularization after the Tl-201 and DISA tests. To assess the functional recovery of dyssynergic segments,
Table 2. Numbers of concordant and discordant segments detected by Tl-201 and DISA $(\mathrm{n}=398)$

DISA SPECT

\begin{tabular}{lcc}
\cline { 2 - 3 } & Viable & Nonviable \\
\hline Tl-201 SPECT & & \\
Viable & $217^{*}$ & 4 \\
Nonviable & 33 & $144^{*}$ \\
\hline
\end{tabular}

${ }^{*}$ Concordant segments.

follow-up echocardiographic studies were performed 1 to 2 months after percutaneous transluminal coronary angioplasty (PTCA) or 3 months after coronary artery bypass grafting $(\mathrm{CABG}))^{3,30}$ The criteria for functional recovery were met if there was an improvement in resting wall motion score by 1 grade or more or an increase in global LVEF of 5\% or greater. Echocardiography was reassessed at least 6 months after revascularization if functional recovery had not been evident from the initial echocardiographic follow-up.

\section{Statistical Analysis}

All results are expressed as mean $\pm \mathrm{SD}$, with categoric data presented as percentages. Comparison of continuous data between groups was performed by use of the Student $t$ test. A $\chi^{2}$ test was used for categoric variables (with the Fisher exact test in smaller sizes). Logistic regression was used to evaluate the statistical significance of DISA and Tl-201 in predicting LV functional recovery. $P<.05$ was considered statistically significant. Statistic data analysis was done with Stata for Windows software (version 8; Stata Press, College Station, Tex).

\section{RESULTS}

\section{Clinical Profiles}

The study cohort consisted of 42 patients. The mean basal plasma glucose level was $101.0 \pm 19.0 \mathrm{mg} / \mathrm{dL}$ (range, $72-120 \mathrm{mg} / \mathrm{dL}$ ) in 31 nondiabetic patients and $222.3 \pm 40.4 \mathrm{mg} / \mathrm{dL}$ (range, $167-303 \mathrm{mg} / \mathrm{dL}$ ) in 11 diabetic patients. For diabetic patients, the mean amount of insulin injected was $7.3 \pm 3.0 \mathrm{IU}$ and the mean plasma glucose level at the time of F-18 FDG injection was $138.2 \pm 28.4 \mathrm{mg} / \mathrm{dL}$ (range, $64-168 \mathrm{mg} / \mathrm{dL}$ ).

All 42 Tc-99m MIBI images obtained in this study were developed with good resolution, but 2 of the 42 F-18 FDG images were not interpretable. These two patients, one of whom had diabetes, had normal glucose 


\section{DISA SPECT}

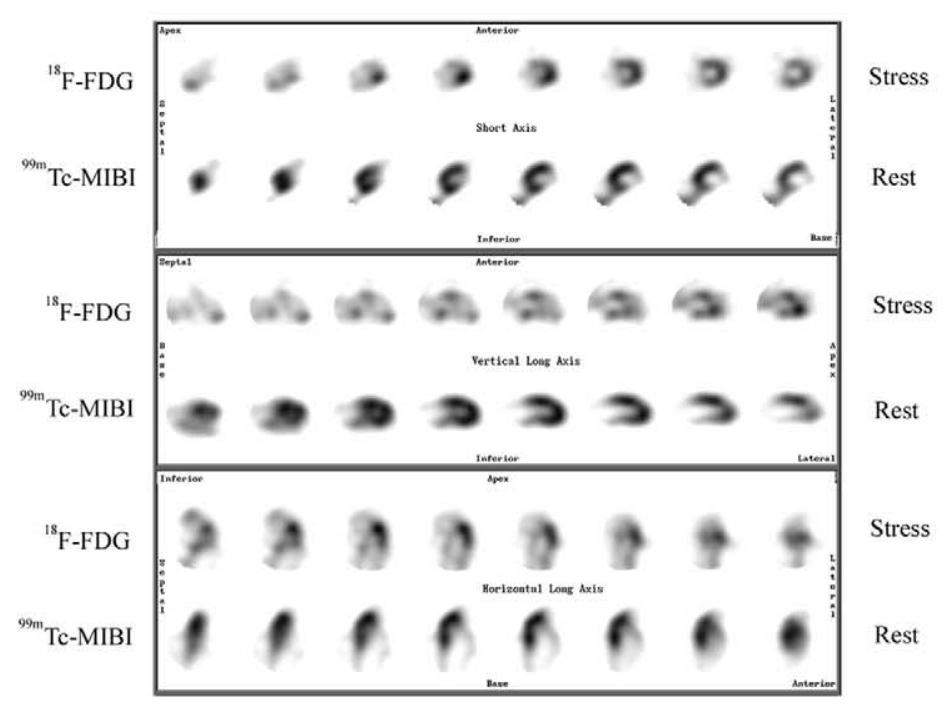

${ }^{201}$ Tl SPECT

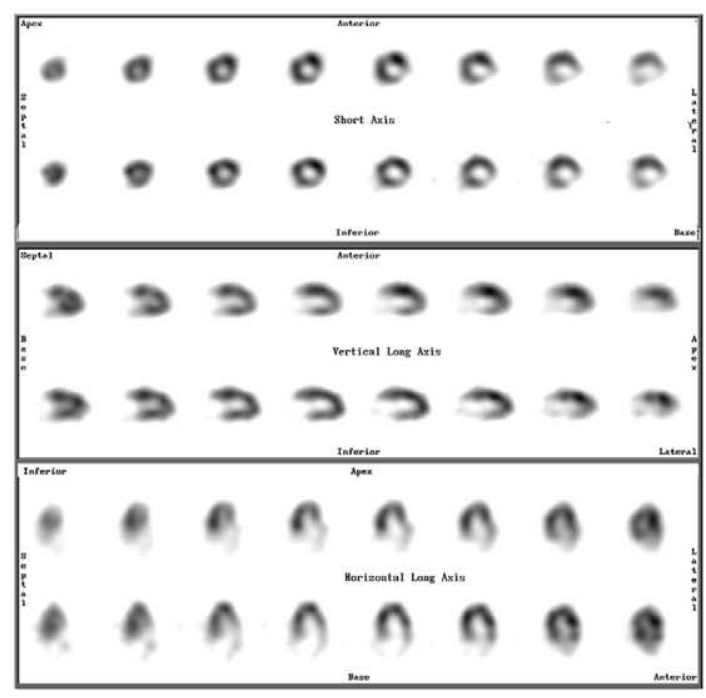

Figure 1. Selected images illustrating discordance between Tl-201 and DISA: DISA-viable but Tl-201-nonviable images in a 70-year-old man with 3-vessel disease. Mismatched perfusion/ metabolism was noted in the inferior and lateral walls on DISA, but fixed perfusion defects were shown in the same areas on stress-reinjection Tl-201 SPECT.

levels at the time of F-18 FDG injection (110 and 122 $\mathrm{mg} / \mathrm{dL}$ ). Their subsequent F-18 FDG PET images turned out to have good resolution nevertheless. The clinical profiles of the remaining 40 patients $(37$ men and 3 women with a mean LVEF of $37.8 \% \pm 15.5 \%$ ) are listed in Table 1.

\section{Comparison of Tl-201 and DISA SPECT}

The results of DISA and Tl-201 SPECT analyzed for all 398 dysfunctional segments (wall motion score $\geq 2$ on resting echocardiography) in 40 patients are listed in Table 2. DISA defined $62.8 \%(250 / 398)$ as viable, whereas Tl-201 defined $55.5 \%(221 / 398)$ as viable. We found that 217 segments were viable by both Tl-201 and DISA and 144 were nonviable by either Tl-201 or DISA. The resulting concordance rate was $90.7 \%$. The viability information obtained from Tc-99m MIBI and Tl-201 was identical. When Tl-201 and DISA results were compared on a per-patient basis, 17 patients $(42.5 \%)$ had completely concordant results, $15(32.5 \%)$ had 1 or 2 discrepant segments, and $8(20 \%)$ had more than 2 discordant segments.

Among the 37 discordant pairs, 33 pairs (89.2\%) were detected as viable by DISA but nonviable by Tl-201 whereas only 4 pairs $(10.8 \%)$ were detected as viable by Tl-201 but nonviable by DISA. Such discrepancy was found to be statistically significant $(P<$ .0001). Representative discordant and concordant images are illustrated in Figures 1 and 2. The distribution of discordant segments is shown in Table 3.

\section{Postrevascularization Follow-up}

Revascularization was performed in 16 patients after Tl-201 and DISA testing either by PTCA (11) or by CABG (5). Of these, 13 (76.5\%) had previous myocardial infarction. The mean follow-up period after revascularization was $9.5 \pm 3.3$ months. Among the 105 dysfunctional segments, 62 segments are considered viable because wall motion improvements were detected during follow-up. The viability results for DISA and Tl-201 are summarized in Table 4. DISA detected 52 of 62 viable segments accurately, whereas Tl-201 detected only 47 . The sensitivity and specificity for viability detection by DISA were $83.9 \%(52 / 62)$ and $37.2 \%$ (16/43), respectively, whereas those for viability detection by Tl-201 were $75.8 \%$ (47/62) and 39.5\% (17/43), respectively. Statistically, DISA viability appears to be a significant predicting factor for functional recovery after revascularization (odds ratio, 3.08; 95\% confidence interval, 1.23-7.70; $P=.014)$, whereas Tl-201 viability does not (odds ratio, 2.05; 95\% confidence interval, $0.88-4.76 ; P=.09)$.

In 14 patients who had 2 or more viable segments according to either DISA or Tl-201, the mean LVEF increased from $38.4 \% \pm 14.7 \%$ (before revascularization) to $47.0 \% \pm 13.0 \%$ (after revascularization) $(P<$ 
DISA SPECT

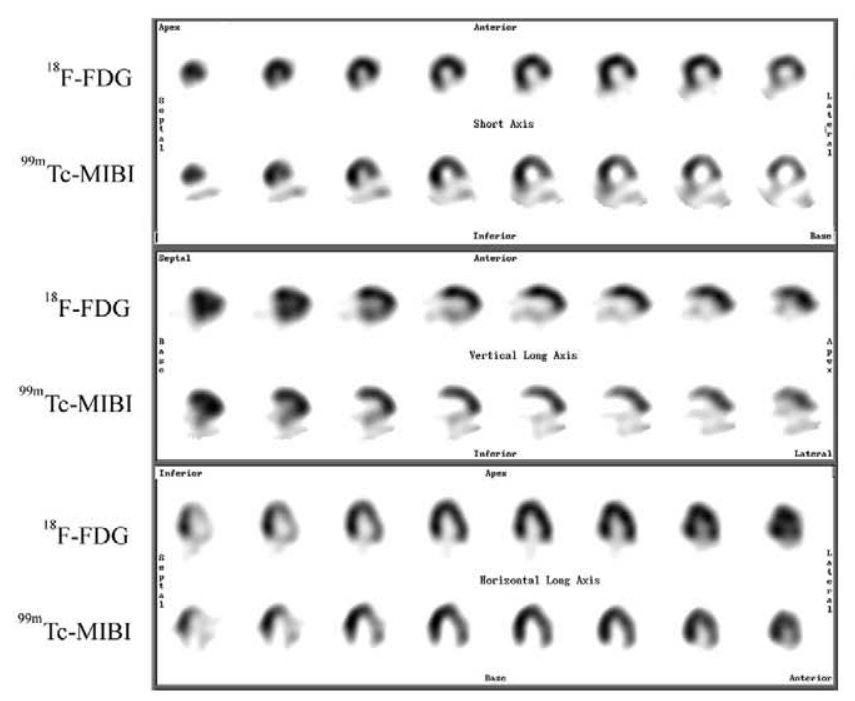

${ }^{201} \mathrm{Tl}$ SPECT

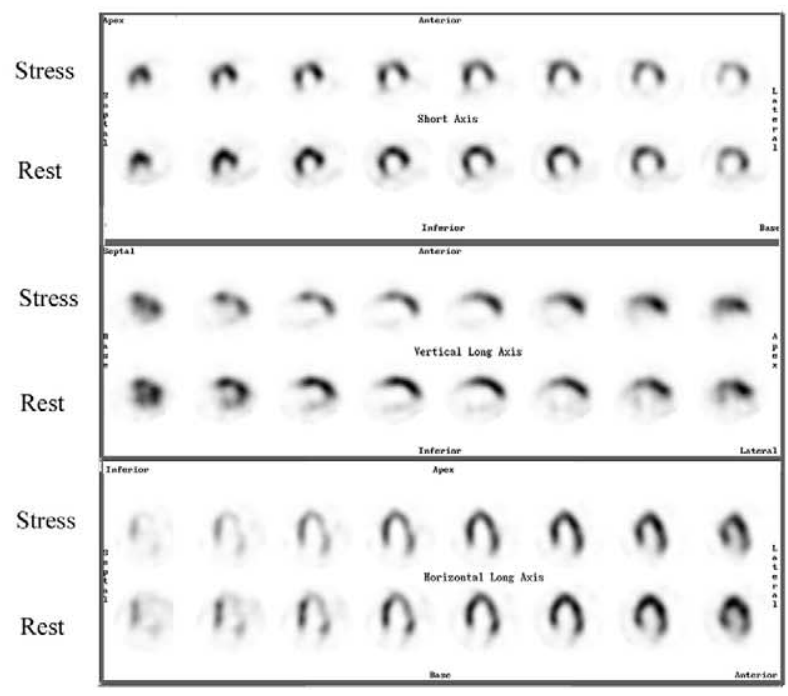

Figure 2. Concordant images of Tl-201 and DISA.

Table 3. Distribution of discordant segments of TI-201 and DISA SPECT

\begin{tabular}{lcc}
\hline & $\begin{array}{c}\text { TI-201-viable/ } \\
\text { DISA- } \\
\text { nonviable } \\
(\mathbf{n}=\mathbf{4})\end{array}$ & $\begin{array}{c}\text { DISA-viable/ } \\
\text { Tl-201- } \\
\text { nonviable } \\
(\mathbf{n}=\mathbf{3 3})\end{array}$ \\
\hline Apex & 0 & $3(9.1 \%)$ \\
Anterior & 0 & $3(9.1 \%)$ \\
Septum & $1(25 \%)$ & $11(33.3 \%)$ \\
$\quad$ Anteroseptal & 1 & 4 \\
Inferoseptal & 0 & 7 \\
Inferior & $1(25 \%)$ & $14(42.4 \%)$ \\
Lateral & $2(50 \%)$ & $2(6.1 \%)$ \\
\hline
\end{tabular}

.001). The NYHA functional class improved in 10 of 14 patients $(71.4 \%)$. There were 6 patients with DISAviable but Tl-201-nonviable segments, and their mean EF increased from $37.7 \% \pm 18.1 \%$ to $45.0 \% \pm 18.7 \%$ $(P<.001)$. On the other hand, the remaining 2 patients with nonviable segments identified by both DISA and Tl-201 testing showed no significant improvement in EF, regional wall motion, and NYHA functional class for 1 year after the intervention (Table 5).

\section{DISCUSSION}

In this study we have assessed myocardial viability in 40 patients using both DISA and Tl-201 stressreinjection SPECT. We have also followed up 16 of these 40 patients by echocardiography to compare the
Table 4. Viability results of DISA and TI-201 in follow-up of 16 patients who underwent revascularization

\begin{tabular}{lcc}
\hline \multirow{2}{*}{$\begin{array}{c}\text { Imaging } \\
\text { method }\end{array}$} & \multicolumn{2}{c}{ Follow-up results } \\
\cline { 2 - 3 } & Viable & Nonviable \\
\hline DISA-viable & 52 & 27 \\
DISA-nonviable & 10 & 16 \\
T1-201-viable & 47 & 26 \\
T1-201-nonviable & 15 & 17 \\
\hline
\end{tabular}

ability of DISA versus Tl-201 to effectively predict postrevascularization functional recovery.

Limited studies have been published on the comparison between F-18 FDG SPECT and Tl-201 SPECT in the evaluation of myocardial viability. ${ }^{5,10,13,31} \mathrm{Al}$ though both F-18 FDG SPECT and Tl-201 SPECT exhibit a high sensitivity for predicting postrevascularization improvement of functional recovery, the specificity of $\mathrm{Tl}-201$ is significantly lower compared with that of F-18 FDG SPECT. ${ }^{32}$ Our results also demonstrate that Tl-201 SPECT can underestimate the extent of myocardial viability, particularly in the inferior region. This discrepancy may partly result from the difference in tracer uptake mechanisms. Tl-201 myocardial uptake reflects cell membrane integrity, ${ }^{33}$ whereas F-18 FDG uptake indicates the overall rates of transmembrane exchange and phosphorylation of glucose. ${ }^{34,35}$ In addition, the inferior 
Table 5. Postrevascularization functional changes in 16 patients who underwent revascularization

\begin{tabular}{|c|c|c|c|c|}
\hline \multirow{2}{*}{$\begin{array}{l}\text { Patient } \\
\text { No. }\end{array}$} & \multirow[b]{2}{*}{ Angiography } & \multirow[b]{2}{*}{ Target vessel } & \multicolumn{2}{|c|}{ Vascular territory viability } \\
\hline & & & Tl-201 SPECT & DISA SPECT \\
\hline 1 & $L M+3 V D$ & RCA & + & + \\
\hline 2 & LAD, RCA & LAD & + & + \\
\hline 3 & LAD, LCX & LAD & - & - \\
\hline 4 & 3VD & CABG (LAD, OM1, PDA) & + & + \\
\hline 5 & $\begin{array}{l}3 V D \text { s/p CABG } \\
\text { SVG } \rightarrow \text { PDA failure }\end{array}$ & $\begin{array}{l}\text { PTCA on SVG } \rightarrow \text { PDA } \\
\text { anastomosis }\end{array}$ & - & + \\
\hline 6 & $3 \mathrm{VD}$ & CABG (LAD, OM1, PDA) & + & + \\
\hline 7 & $3 \mathrm{VD}$ & LAD, LCX & $\begin{array}{l}\text { LAD+ } \\
\text { LCX- }\end{array}$ & $\begin{array}{l}+ \\
-\end{array}$ \\
\hline 8 & LCX, RCA & $\mathrm{RCA}$ & - & + \\
\hline 9 & $\mathrm{LM}, \mathrm{LAD}, \mathrm{RCA}$ & CABG (LAD, D1, PDA) & $\begin{array}{l}\mathrm{LAD}+ \\
\mathrm{RCA}-\end{array}$ & $\begin{array}{l}+ \\
+\end{array}$ \\
\hline 10 & LAD & LAD & - & - \\
\hline 11 & $\begin{array}{l}3 V D \text { s/p CABG } \\
\text { SVG } \rightarrow \text { LAD 60\%, } \\
\text { OM, RCA } 100 \%\end{array}$ & Re-CABG (LAD, OM, RCA) & $\begin{array}{l}\text { LAD+ } \\
\text { LCX- } \\
\text { RCA- }\end{array}$ & $\begin{array}{l}+ \\
+ \\
+\end{array}$ \\
\hline 12 & $3 V D$ & RCA & - & + \\
\hline 13 & LAD, RCA & LAD & + & + \\
\hline 14 & $3 \mathrm{VD}$ & CABG (LAD, OM1, PDA) & + & + \\
\hline 15 & LCX, OM2, RCA & LCX, OM1 & + & + \\
\hline 16 & $\begin{array}{l}\text { 3VD s/p CABG, } \\
\text { retrograde flow of } \\
\text { SVG, D1, OM1, PLA }\end{array}$ & $\mathrm{LAD}$ & - & + \\
\hline
\end{tabular}

$L M$, Left main; VD, vessel disease; $L A D$, left anterior descending artery; $L C X$, left circumflex artery; $R C A$, right coronary artery; $D 1$, first diagonal branch; $O M$, obtuse marginal branch; PDA, posterior descending artery; PLA, posterior lateral artery; SVG, saphenous venous graft; + , viable; -, nonviable; $s / p$, status post.

wall is one of the most severely attenuated regions, and greater attenuation of Tl-201 relative to F-18 FDG in the inferior segments may result.

Four segments in four patients were diagnosed as Tl-201-viable but DISA-nonviable. In addition to the aforementioned difference in tracer uptake mechanisms, this result may be attributed to the fact that Tl-201 is more sensitive than F-18 FDG PET in detecting segments containing less than $75 \%$ viable myocytes, as suggested by Baumgartner et $\mathrm{al}^{36}$ in a histopathologic correlation study. Because none of these 4 patients had undergone revascularization, the clinical implication and relevance of the test results to these 4 segments remain to be determined.

There is a known pitfall when using DISA in patients with diabetes. Significant numbers of diabetic patients exhibit poor myocardium F-18 FDG uptake after the administration of soluble insulin. The use of the euglycemic hyperinsulinemic clamp has been reported to yield excellent metabolic images, but it can also increase the cost and complexity of DISA signif- icantly. ${ }^{14,15}$ Recently, F-18 FDG SPECT after oral administration of the nicotinic acid derivative acipimox was shown to be a safe approach and to result in good image quality for patients with diabetes. ${ }^{37,38}$ If such a procedure is applicable to our studies, the preparation of DISA for diabetic patients can be further simplified accordingly.

Although Sandler and colleagues ${ }^{14,15}$ have proposed that good image quality can be obtained from DISA, the F-18 FDG SPECT images obtained for 2 of 42 sample patients in this study were too poor to be interpretable. The subsequent F-18 FDG PET images taken for these 2 patients did exhibit good resolution. Indeed, it has previously been reported by Hasegawa et $\mathrm{al}^{39}$ that images obtained by use of ultrahigh-energy SPECT can exhibit poor resolution compared with those obtained by PET.

In general, the preparation procedures for DISA are more complicated than those for Tl-201 SPECT. Furthermore, F-18 FDG is an expensive reagent and must be provided by a nearby cyclotron because of its 


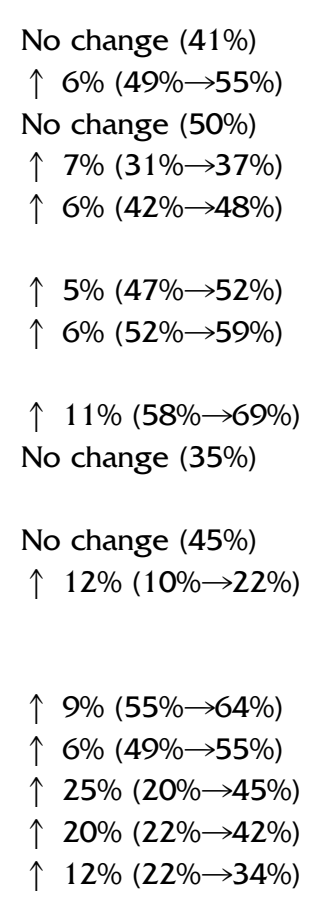

$$
\begin{gathered}
\mathrm{III} \rightarrow \mathrm{II} \\
\mathrm{II} \rightarrow \mathrm{II} \\
\mathrm{II} \rightarrow \mathrm{II} \\
\mathrm{III} \rightarrow \mathrm{II} \\
\mathrm{III} \rightarrow \mathrm{II} \\
\\
\mathrm{II} \rightarrow \mathrm{II} \\
\mathrm{II} \rightarrow \mathrm{I} \\
\mathrm{II} \rightarrow \mathrm{I} \\
\mathrm{III} \rightarrow \mathrm{III} \\
\\
\mathrm{II} \rightarrow \mathrm{II} \\
\mathrm{IV} \rightarrow \mathrm{III} \\
\\
\\
\mathrm{II} \rightarrow \mathrm{II} \\
\mathrm{II} \rightarrow \mathrm{I} \\
\mathrm{IV} \rightarrow \mathrm{II} \\
\mathrm{IV} \rightarrow \mathrm{II} \\
\mathrm{III} \rightarrow \mathrm{II}
\end{gathered}
$$$$
\begin{aligned}
& \text { No change } \\
& \text { Improved in 3-vessel territories }
\end{aligned}
$$

short half-life. Although the equipment for DISA is more costly than that of Tl-201 SPECT, the actual cost incurred from using Tl-201 SPECT may increase because a third acquisition of reinjection images is often needed. On the other hand, DISA requires only a single SPECT acquisition with shorter image acquisition time. We suggest that the cost-effectiveness of DISA in comparison with Tl-201 SPECT needs to be further studied and fully evaluated. ${ }^{40,41}$

\section{Study Limitations}

No attenuation corrections were used for either DISA or Tl-201 SPECT in this study. The difference in attenuation by diaphragm between Tc-99m MIBI and F-18 FDG has been reported to be a potential source of false-positive results in the inferior myocardium in DISA. ${ }^{14,15,17}$ Furthermore, the inferior wall is one of the most severely attenuated regions of Tl-201 and may erroneously yield a false-negative Tl-201 result without attenuation correction. In this study most discrepancies of DISA and Tl-201 were observed in the inferior wall.

No follow-up angiography was performed after revascularization to access coronary/graft patency for the 16 patients who received PTCA or CABG. Coronary/ graft occlusion might have prohibited functional recovery of segments diagnosed as viable by DISA or Tl-201.

Patients with severe LV dysfunction are the most relevant group of patients whose diagnosed viability results may play a crucial role in management decisions. Unfortunately, only a relatively small number of these patients were included in this study. Nevertheless, we believe that our results will be reinforced by future study with a large sample of patients who have severe LV dysfunction.

\section{Conclusion}

Our study suggests that DISA viability provides a more accurate prediction of postrevascularization functional recovery than Tl-201 viability. Given the small number of patients who underwent revascularization in this study (16), the superiority of DISA over Tl-201 in detecting myocardial viability may be firmly established by further study on a large scale of patients with profound LV dysfunction. 


\section{Acknowledgment}

The authors have indicated they have no financial conflicts of interest.

\section{References}

1. Gheorghiade M, Bonow RO. Chronic heart failure in the United States: a manifestation of coronary artery disease. Circulation 1998;97:282-9.

2. van den Berg EK Jr, Popma JJ, Dehmer GJ, Snow FR, Lewis SA, Vetrovec GW, et al. Reversible segmental left ventricular dysfunction after coronary angioplasty. Circulation 1990;81:1210-6.

3. Huang PJ, Lin LC, Yen RF, Ho YL, Wu CC, Hsu KL, et al. Accuracy of biphasic response, sustained improvement and worsening during dobutamine echocardiography in predicting recovery of myocardial dysfunction after revascularization: comparison with simultaneous thallium-201 reinjection SPECT. Ultrasound Med Biol 2001;27:925-31.

4. Bonow RO. Identification of viable myocardium. Circulation 1996;94:2674-80.

5. Bax JJ, Wijns W, Cornel JH, Visser FC, Boersma E, Fioretti PM. Accuracy of currently available techniques for prediction of functional recovery after revascularization. J Am Coll Cardiol 1997;30:1451-60.

6. Eitzman D, al-Aouar Z, Kanter HL, vom Dahl J, Kirsh M, Deeb GM, et al. Clinical outcome of patients with advanced coronary artery disease after viability studies with positron emission tomography. J Am Coll Cardiol 1992;20:559-65.

7. Di Carli M, Davidson M, Little R, Khanna S, Mody FV, Brunken $\mathrm{RC}$, et al. Value of metabolic imaging with positron emission tomography for evaluating prognosis in patients with coronary artery disease and left ventricle dysfunction. Am J Cardiol 1994; 73:527-33.

8. Tillisch J, Brunken R, Marshall R, Schwaiger M, Mandelkern M, Phelps M, et al. Reversibility of cardiac wall-motion abnormalities predicted by positron emission tomography. N Engl J Med 1986; 314:884-8.

9. Conti PS, Keppler JS, Halls JM. Positron emission tomography: a financial and operational analysis. AJR Am J Roentgenol 1994; 162:1279-86.

10. Burt RW, Perkins OW, Oppenheim BE, Schauwecker DS, Stein L, Wellman HN, et al. Direct comparison of fluorine-18-FDG SPECT, fluorine-18-FDG PET and rest thallium SPECT for detection of myocardial viability. J Nucl Med 1995;36:176-9.

11. Bax JJ, Visser FC, van Lingen A, Huitink JM, Kamp O, van Leeuwen GR, et al. Feasibility of assessing regional myocardial uptake of ${ }^{18} \mathrm{~F}$-fluorodeoxyglucose using single photon emission computed tomography. Eur Heart J 1993;14:1675-82.

12. Martin WH, Delbeke D, Patton JA, Hendrix B, Weinfeld Z, Ohana I, et al. FDG-SPECT: correlation with FDG-PET. J Nucl Med 1995;36:988-95.

13. Bax JJ, Visser FC, Blanksma PK, Veening MA, Tan ES, Willemsen TM, et al. Comparison of myocardial uptake of ${ }^{18} \mathrm{~F}$-fluorodeoxyglucose imaged with PET and SPECT in dyssynergic myocardium. J Nucl Med 1996;37:1631-6.

14. Delbeke D, Videlefsky S, Patton JA, Campbell MG, Martin WH, Ohana I, et al. Rest myocardial perfusion/metabolism imaging using simultaneous dual-isotope acquisition SPECT with technetium-99m-MIBI/fluorine-18-FDG. J Nucl Med 1995;36:2110-9.

15. Sandler MP, Videlefsky S, Delbeke D, Patton JA, Meyerowitz C, Martin WH, et al. Evaluation of myocardial ischemia using a rest metabolism/stress perfusion protocol with fluorine-18 deoxyglucose/technetium-99m MIBI and dual-isotope simultaneous-acqui- sition single-photon-emission computed tomography. J Am Coll Cardiol 1995;26:870-8.

16. Schoder H, Campisi R, Ohtake T, Hoh CK, Moon DH, Czernin J, et al. Blood flow-metabolism imaging with positron emission tomography in patients with diabetes mellitus for the assessment of reversible left ventricular contractile dysfunction. J Am Coll Cardiol 1999;33:1328-37.

17. Sandler MP, Bax JJ, Patton JA, Visser FC, Martin WH, Wijns W. Fluorine-18 deoxyglucose cardiac imaging using a modified scintillation camera. J Nucl Med 1998;39:2035-43.

18. Huang PJ, Chieng PU, Lee YT, Chiang FT, Tseng YZ, Liau CS, et al. Exercise thallium-201 tomographic scintigraphy in the diagnosis of coronary artery disease: emphasis on the effect of exercise level. J Formos Med Assoc 1992;91:1096-101.

19. Ho FM, Huang PJ, Liau CS, Lee FK, Chieng PU, Su CT. Dobutamine stress echocardiography compared with dipyridamole thallium-201 single-photon emission computed tomography in detecting coronary artery disease. Eur Heart J 1995;16:570-5.

20. Huang PJ, Yen RF, Chieng PU, Chen ML, Su CT. Do $\beta$-blockers affect the diagnostic sensitivity of dobutamine thallium-201 single photon emission computed tomographic imaging? J Nucl Cardiol 1998;5:34-9.

21. Cerqueira MD, Weissman NJ, Dilsizian V, Jacobs AK, Kaul S, Laskey WK, et al. Standardized myocardial segmentation and nomenclature for tomographic imaging of the heart: a statement for healthcare professionals from the Cardiac Imaging Committee of the Council on Clinical Cardiology of the American Heart Association. Circulation 2002;105:539-42.

22. Chan RKM, Lee KJ, Calafiore P, Berlangieri SU, McKay WJ, Tonkin AM. Comparison of dobutamine echocardiography and positron emission tomography in patients with chronic ischemic left ventricular dysfunction. J Am Coll Cardiol 1996;27:1601-7.

23. vom Dahl J, Altehoefer C, Sheehan FH, Buechin P, Schulz G, Schwarz ER, et al. Effect of myocardial viability assessed by technetium-99m-sestamibi SPECT and fluorine-18-FDG PET on clinical outcome in coronary artery disease. J Nucl Med 1997;38: $742-8$.

24. Rambaldi R, Poldermans D, Bax JJ, Boersma E, Valkema R, Elhendy A, et al. Dobutamine stress echocardiography and technetium-99m-tetrofosmin/fluorine-18-fluorodeoxyglucose singlephoton emission computed tomography and influence of resting ejection fraction to assess myocardial viability in patients with severe left ventricular dysfunction and healed myocardial infarction. Am J Cardiol 1999;84:130-4.

25. Dilsizian V, Rocco TP, Freedman NMT, Leon MB, Bonow RO. Enhanced detection ischemic but viable myocardium by the reinjection of thallium after stress-redistribution imaging. N Engl J Med 1990;323:141-6.

26. Takahashi N, Tamaki N, Tadamura E, Kawamoto M, Torizuka T, Yonekura Y, et al. Combined assessment of regional perfusion and wall motion in patients with coronary artery disease with technetium 99m tetrofosmin. J Nucl Cardiol 1994;1:29-38.

27. Dilsizian V, Freedman NMT, Bacharach SL, Perrone-Filardi P, Bonow RO. Regional thallium uptake in irreversible defects: magnitude of change in thallium activity after reinjection distinguishes viable from nonviable myocardium. Circulation 1992;85: $627-34$.

28. Schiller NB, Shah PM, Crawford M, DeMaria A, Devereux R, Feigenbaum H, et al. Recommendations for quantitation of the left ventricle by two-dimensional echocardiography. American Society of Echocardiography Committee on Standards, Subcommittee on Quantitation of Two-Dimensional Echocardiograms. J Am Soc Echocardiogr 1989;2:358-67. 
29. Ho YL, Lin LC, Yen RF, Wu CC, Chen MF, Huang PJ. Significance of dobutamine-induced ST-segment elevation and T-wave pseudonormalization in patients with Q-wave myocardial infarction: simultaneous evaluation by dobutamine stress echocardiography and thallium-201 SPECT. Am J Cardiol 1999;84:125-9.

30. Sciagra R, Bisi G, Santoro GM, Zerauschek F, Sestini S, Pedenovi $\mathrm{P}$, et al. Comparison of baseline-nitrate technetium- $99 \mathrm{~m}$ sestamibi with rest-redistribution thallium-201 tomography in detecting viable hibernating myocardium and predicting post-revascularization recovery. J Am Coll Cardiol 1997;30:384-91.

31. Bax JJ, Cornel JH, Visser FC, Fioretti PM, van Lingen A, Reijs AE, et al. Prediction of recovery of myocardial dysfunction after revascularization: comparison of fluorine-18fluorodeoxyglucose/thallium-201 SPECT, thallium-201 stressreinjection SPECT and dobutamine echocardiography. J Am Coll Cardiol 1996;28:558-64.

32. Srinivasan G, Kitsiou AN, Bacharach SL, Bartlett ML, MillerDavis C, Dilsizian V. ${ }^{18} \mathrm{~F}$-Deoxyglucose SPECT: can it replace PET and thallium SPECT for the assessment of myocardial viability? Circulation 1998;97:843-50.

33. Pohost GM, Zir LM, Morre RH, McKusick KA, Guiney TE, Beller GA. Differentiation of transiently ischemic from infarcted myocardium by serial imaging after a single dose of thallium-201. Circulation 1977;55:294-302.

34. Wheeler TJ. Translocation of glucose transporters in response to anoxia in heart. J Biol Chem 1988;263:19447-54.
35. Young LH, Rendu Y, Russell R, Hu X, Caplan M, Ren J, et al. Low-flow ischemia leads to translocation of canine heart GLUT-4 and GLUT-1 glucose transporters to the sarcolemma in vivo. Circulation 1997;95:415-22.

36. Baumgartner H, Porenta G, Lau YK, Wutte M, Klaar U, Mehrabi $\mathrm{M}$, et al. Assessment of myocardial viability by dobutamine echocardiography, positron emission tomography and thallium201 SPECT. Correlation with histopathology in explanted hearts. J Am Coll Cardiol 1998;32:1701-8.

37. Schinkel AFL, Bax JJ, Valkema R, Elhendy A, van Domburg RT, Vourvouri EC, et al. Effect of diabetes mellitus on myocardial ${ }^{18}$ F-FDG SPECT using acipimox for the assessment of myocardial viability. J Nucl Med 2003;44:877-83.

38. Kam BL, Valkema R, Poldermans D, Bax JJ, Reijs AE, Rambaldi $\mathrm{R}$, et al. Feasibility and image quality of dual-isotope SPECT using ${ }^{18} \mathrm{~F}$-FDG and ${ }^{99 \mathrm{~m}} \mathrm{Tc}$-tetrofosmin after acipimox administration. J Nucl Med 2003;44:140-5.

39. Hasegawa S, Uehara T, Yamaguchi H, Fujino K, Kusuoka H, Hori $\mathrm{M}$, et al. Validity of ${ }^{18} \mathrm{~F}$-fluorodeoxyglucose imaging with a dual-head coincidence gamma camera for detection of myocardial viability. J Nucl Med 1999;40:1884-92.

40. Udelson JE. Steps forward in the assessment of myocardial viability in left ventricular dysfunction. Circulation 1998;97:833-8.

41. Beller GA, Zaret BL. Contribution of nuclear cardiology to diagnosis and prognosis of patients with coronary artery disease. Circulation 2000;101:1465-78. 